\title{
Concepción metodológica del diseño y rediseño de carreras en la Universidad Internacional SEK Ecuador
}

\section{Methodological conception of design and redesign of careers at SEK International University Ecuador}

Enrique Aurelio Barrios Queipo. PhD

Alfonso Algora Buenafé

Nadia Margarita Rodríguez Jiménez

Universidad Internacional SEK Ecuador, Ecuador

Autor para correspondencia: enrique.barrios@uisek.edu.ec

Fecha de recepción: 30 de Agosto de 2016 - Fecha de aceptación: 25 de Octubre de 2016

Resumen: Ante los desafíos en la formación de profesionales, la mejora de la oferta formativa de las carreras universitarias, a partir de su diseño y rediseño curricular sistemático, constituye uno de los retos de mayor prioridad en la Educación Superior ecuatoriana. Para lograr este propósito y superar las limitaciones que se acumulaban en la necesaria integración que debe lograrse entre las demandas del Consejo Educación Superior, las aspiraciones del Modelo Educativo de la Universidad Internacional SEK y la concreción de cada una de sus carreras, se crea un Comité Curricular cuya misión es la de interpretar esta integración y elaborar una concepción metodológica institucional que favoreciera en las acciones de diseño y rediseño que desarrollan las carreras, la coherencia entre lo general, lo particular y lo singular de estos procesos. El artículo que se presenta sintetiza los fundamentos de los puntos de vista que sustentan cómo desarrollar estos procesos de manera que se alcance una nueva oferta formativa de interés y al mismo tiempo tenga un carácter de futuro para el desarrollo socioeconómico contextual. Se aplicó el método sistémico - estructural - funcional, develándose que de las relaciones entre el proceso profesional, el proceso de formación profesional y el proceso de pertinencia universitaria, pueden condensar lo pertinente de una carrera universitaria.

Palabras claves: profesión; proceso profesional; pertinencia universitaria; proceso formativo; diseño y rediseño de carreras universitarias

Abstract: Given the challenges in the training of professionals, improving training offer university degrees, from its design and systematic curriculum redesign, it is one of the challenges of higher priority in the Ecuadorian Higher Education. To achieve this purpose and overcome the limitations that accumulated in the necessary integration to be achieved between the demands of the Council Higher Education, the aspirations of the educational model of the International University SEK and the realization of each of their careers, a committee created curricular whose mission is to interpret this integration and develop an institutional methodological conception that favored in the design and redesign actions that develop careers, consistency between the general, the particular and the singular of these processes. In the article "methodological concept design racing in the UISEK_Ecuador the fundamentals of the views that support how to develop these processes so that a new training offer interest is achieved and at the same time have a character for the future of synthesized contextual socioeconomic development. Structural systemic functional method was 
applied, develándose that relations between the professional process, the training process and the process of university relevance, can condense pertinent to college.

Key words: profession; professional process; university relevance; training process; design and redesign of university courses

\section{Introducción}

Las universidades ecuatorianas, en relación con los diseños y rediseños de sus carreras, sistematizan diversos procesos institucionales que intentan hacer realidad la integración entre los desafíos y perspectivas del país, las aspiraciones de desarrollo de cada universidad y la práctica que realizan sus carreras con este propósito.

Los resultados e impacto del proceder que han ejecutado los colectivos de carreras en la Universidad Internacional SEK_Quito (UISEK), en los últimos tres años, en atención a los procesos de aprobación de programas y de acreditación de las carreras por parte de las autoridades gubernamentales competentes, permitieron demostrar que se hacía necesaria la toma de decisiones para desarrollar nuevas alternativas institucionales de manera de garantizar que sus comité curriculares de carreras pudieran satisfacer las exigencias de los diseños y rediseños de carreras.

Agravaba la situación expresada, conocer algunas manifestaciones expresadas por actores de las diferentes carreras (Decanos de Facultades, coordinadores de carreras, profesores participantes), los que referían que la aplicación de la metodología establecida por el Consejo de Educación Superior ecuatoriano para evidenciar documentalmente los nuevos diseños y rediseños de carreras, en tanto que facilitaba hilvanar un grupo de decisiones curriculares mediante respuestas a un complejo cuestionario establecido y de obligatorio cumplimiento, igualmente no les ofrecía toda la orientación necesaria para que dichos diseños estuvieran a la altura de las demandas establecidas. Esta situación venía comprometiendo la calidad de los diseños y rediseño y su no aprobación oficial.

En relación con lo anterior, también los autores del presente artículo reconocen múltiples manifestaciones de expertos de las Comisiones ocasionales de carreras, de especialistas que participan en las REDES universitarias y de los informes que limitan la aprobación de los programas de carreras, que hacían suponer, que la metodología establecida por el Consejo de Educación Superior ecuatoriano para elaborar dichos programas, no cumplía con su objetivo.

Ante la situación problémica planteada, referida a la no contribución de una metodología establecida para lograr un propósito, de acuerdo con Valle (2010), pudo aceptarse que científicamente es posible que un "el instrumento (en este caso la metodología) no se corresponda con su sustento teórico, o que el sustento teórico no satisfaga la viabilidad de dicho instrumento". En tal sentido fue posible profundizar en ambos (la teoría y su instrumento) y reflexionar acerca de la solución de dicha situación.

Los autores del presente trabajo, al analizar que la concepción teórica que sustenta la metodología de diseño y rediseños de carreras universitarias en Ecuador y la propia metodología, dado la relación de ambos con los documentos que la originan (Leyes, Normativas), tienen cierto 
grado de fundamentación para su justificación, pero no logran expresar dicha fundamentación para la acción.

La idea de la concepción teórica y su instrumento asociado es la de garantizar carreras universitarias con apego a al principio de la pertinencia. Eso es válido.

El principio de la pertinencia, tal y como lo expresa la Ley Orgánica de la Educación Superior (LOES, 2010), exhorta a que el proceso de diseño y rediseño de las carreras, responda a las expectativas y necesidades de la sociedad, a la planificación nacional, y al régimen de desarrollo, a la prospectiva de desarrollo científico, humanístico y tecnológico mundial, y a la diversidad cultural. Desde esta perspectiva, dicho proceso está dentro de un marco lógico que favorece conducirlo al cumplimiento de la primera Ley de Universidades "El vínculo permanente de la universidad con su medio, con la sociedad” (Álvarez, 2004).

Estas intenciones son positivas para alcanzar los resultados en la universidad, no obstante consideramos que un principio o su cumplimiento no son suficiente para que un proceso logre determinado objetivo. Es evidente que se precise si el resultado del proceso (en relación con la magnitud del propio objetivo, del contenido empleado o del método seleccionado), responde a la solución del problema que lo originó, lo que en el caso del diseño y rediseño de las carreras en la UISEK y de otras instituciones universitarias no se lograba. Es decir, se intentaba cumplimentar una metodología con un contenido que no reflejaba la cultura curricular que podía satisfacer la formación del estudiantado con la pertinencia universitaria correspondiente.

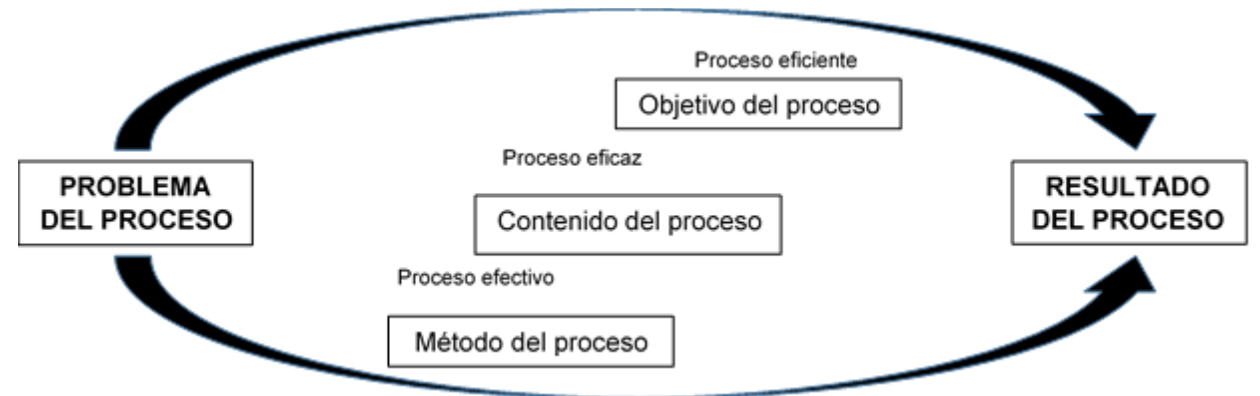

Figura 1.- Relaciones de evaluación del resultado de un proceso respecto al problema que lo origina, cuando se tienen en cuenta el objetivo, el contenido y el método del proceso.

Somos consecuentes con las ideas de Fuentes (1998), quien considera que el resultado de un proceso respecto al problema que lo origina tiene, a partir del objetivo su satisfacción, o sea es un proceso eficiente; a partir del contenido es un proceso eficaz; y a partir del método, es un proceso efectivo (Figura 1).

Los autores de este trabajo al profundizar en el estudio del proceso de diseño y rediseño de las carreras, asumen que el principio de pertinencia crea el marco lógico para lograr su eficiencia, eficacia y efectividad, pero en la revisión de la situación problémica por la cual no se satisface la mejora de los programas de carreras, se puede interpretar que el objetivo a que se aspira con la metodología está fundamentado, pero que el contenido con el cual se satisfacen sus actividades no resuelve el problema. Es un proceso poco eficaz. 
El contenido con el cual las carreras satisfacen las actividades de la metodología de su diseño y rediseño es una determinación de las carreras, no es parte de la metodología. La metodología orienta cumplir las actividades.

Esto trae aparejado que la universidad contextualiza una metodología cuya concepción, basada en el principio de la pertinencia es adecuada, pero no logra que desde las carreras se satisfaga con el contenido. He allí un resultado cuyo contenido no resuelve el problema que lo origina. Se ratifica la poca eficacia del proceso, se concluye en la no existencia de una concepción institucional que pudiera favorecer la precisión de un contenido para contribuir al desarrollo eficaz de la metodología de diseño y rediseño de carreras.

\section{Desarrollo}

Desde el punto de vista teórico, aplicar una metodología general del Consejo Educación Superior, sin una concepción que asuma lo general, lo particular y lo singular del contexto, no permite reflejar su potencialidad como instrumento de esa teoría. En tal sentido, la metodología que aplican las universidades ecuatorianas para el proceso de diseño y rediseño de sus carreras sin una concepción de universidad no facilita su eficacia y entonces no logra enunciar su oferta formativa con los requerimientos de dicha metodología. He allí la limitación de la metodología señalada.

De acuerdo con lo establecido en la Ley Orgánica de Educación Superior en Ecuador (LOES, 2010), las instituciones de Educación Superior articularán su oferta formativa a la demanda académica, a las necesidades de desarrollo y tendencias del mercado laboral local, regional y nacional; a la innovación y diversificación de profesiones; a las tendencias demográficas; a la vinculación con la estructura productiva actual y potencial y a las políticas nacionales de ciencia y tecnología. Ello tiene que ver con el principio de la pertinencia.

De lo anterior se deriva que la formación de los profesionales está sometida a las demandas de varios aspectos de rigor. Una de las vías de mayor impacto en la atención a estas demandas, para la retroalimentación del proceso formativo universitario, son los procesos de diseño y rediseños de carreras mediante proceso de innovación.

Un diagnóstico realizado por los autores del presente trabajo, como parte de sus funciones institucionales en la UISEK, sobre el estado del rediseño de sus carreras durante los últimos dos años académicos, facilitó develar que dicha aspiración estaba limitada por la falta de precisión del contenido, a través de la cual, sus actores ejercían sus labores de rediseño.

En la búsqueda de las posibles soluciones aplicadas para enfrentar la situación presentada, se identificaron diversos hechos sobre el contenido utilizado en el proceso de rediseño de las carreras constatadas, a partir de la revisión de diversas fuentes de información científicas. Ello facilitó identificar lo siguiente:

$\checkmark$ Los documentos de rediseño de carreras no develan con claridad su apego al cumplimiento del principio de pertinencia. Ello compromete que la propuesta constituya una declaración de 
ajuste a las nuevas expectativas de los profesionales universitarios que necesita el desarrollo socioeconómico del país.

$\checkmark$ En lo referido al macrodiseño curricular de las carreras, se observan incoherencias entre la generalidad de la propuesta que debe satisfacer determinadas normativas del sistema universitario y la singularidad del modelo que aplica la UISEK.

De esta manera, el rediseño de las carreras aplicado, no satisface la concreción, con carácter perspectivo, de las características más esenciales del egresado exigidas por el contexto socioeconómico analizado. Así, la innovación del proceso formativo desarrollada en los diseños antecedentes, ha dejado de ser un proceso que transforme mediante sus cambios de esencia, la práctica formativa en la UISEK.

Se corrobora con lo expresado, que la relación entre la innovación del proceso formativo, ejecutada mediante el proceso de rediseño de las carreras, respecto al principio de la pertinencia que lo delimita, muestra falta de correspondencia y ello limita que las nuevas cualidades esperadas de esta relación (cualidades que expresan la sistematización de diseño de las carreras para la mejora del proceso formativo y de retroalimentación intencionada de las necesidades contextuales para esa formación), garanticen un rediseño pertinente. (Figura 2).

En este trabajo se asume la innovación del proceso formativo como “...un proceso creador, mediante el cual se producen cambios de esencia en la concepción del profesional y la cultura que lo sustenta, que tiene un carácter pedagógico, así como de la malla de estudio y la estructura y organización de los componentes de formación, que se concreta en la estrategia de la carrera, que tiene un carácter didáctico. Innovar implica definir bases o criterios de innovación, sistematizar, secuenciar, controlar, retroalimentar para que su resultado satisfaga la necesidad de su elaboración”. (Barrios y Delgado, 2015).

Igualmente se entenderá como pertinencia de carreras, a la articulación de la oferta formativa con el régimen constitucional del Buen Vivir, el Plan Nacional de Desarrollo, los planes regionales y locales, los requerimientos sociales en cada nivel territorial y las corrientes internacionales científicas y humanísticas de pensamiento. (Reglamento de Régimen Académico CES Ecuador, 2013).

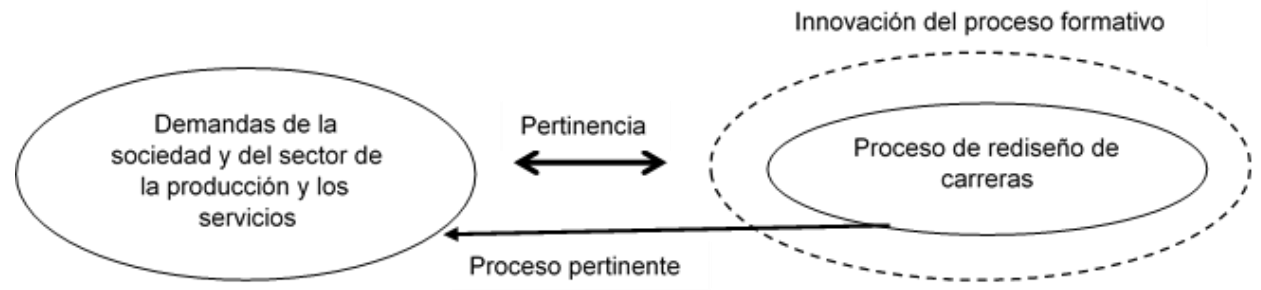

Figura 2.- Representación gráfica del principio de pertinencia con la relación entre la innovación del proceso formativo, a través del rediseño de las carreras, y las demandas del contexto social.

La búsqueda de la correspondencia entre la innovación del proceso formativo, ejecutada mediante el proceso de diseño y rediseño de las carreras, respecto al principio de la pertinencia que lo delimita, ha sido abordado por innumerables autores entre otros destacan Coll (1987), Arbizu (1998), Álvarez (2004), Portuondo y Barrios (2004), Fuentes (2010), los que significan y coinciden en que este es un proceso perteneciente al diseño curricular, parte de su 
retroalimentación permanente para que el currículo responda a las necesidades de la formación profesional. Es la génesis de la tríada que puede ser estructurada por: el proceso profesional; el proceso formativo y el proceso de pertinencia y que los autores del presente trabajo consideran imprescindible para ejecutar con éxito el proceso señalado.

En general, este análisis teórico subraya que es imprescindible que cada carrera profundice en el conocimiento de la profesión, sus problemas profesionales y su proceso profesional. Se unen a ello precisar las demandas de la sociedad en cuestión. Ese es el punto de partida, allí está el origen de la pertinencia de una carrera. Pero ha sido muy complejo que las universidades interpreten que esa es la pertinencia en su carácter genérico, igualmente, que esa pertinencia debe ser derivada a su carácter particular y singular.

Atender el principio de pertinencia, es enlazar lo general que aspira la Educación Superior ecuatoriana con lo particular que pretende cada universidad y con lo singular de sus carreras. Es decir, lo particular refleja el modelo formativo que asume cada universidad, es lo que la distingue del resto de las universidades. De la misma manera, lo singular de las carreras lleva implícito la concreción del proceso formativo, sintetiza cómo pretende la carrera formar a su alumnos.

Establecer esta relación en el diseño de carreras, a partir de la guía que promueve la metodología para su diseño y rediseños consecutivos, puede ser explicada en el sistema de relaciones que se verifican entre el proceso profesional - necesidad social, el proceso formativo y el proceso de pertinencia; que tiene su síntesis teórica en el alcance de un proceso pertinente (Figura 3).

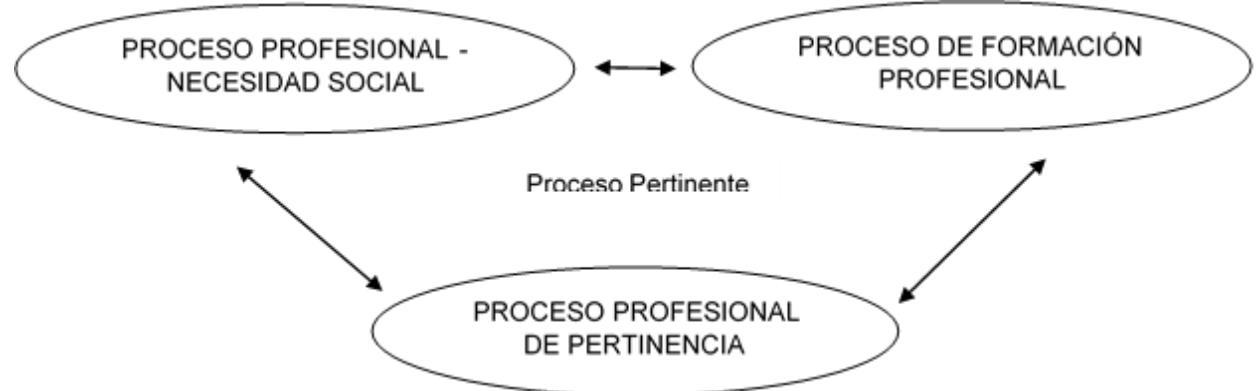

Figura 3.- Subsistemas que en su relaciones condicionan el alcance del diseño y rediseño de carreras en la búsqueda de su cualidad como proceso pertinente.

Bajo esta tríada, se concibe en la UISEK, la determinación del contenido que satisface la metodología para el proceso de diseño y rediseño de las carreras. Es una concepción que favorece hacer una interpretación de lo que se requiere valorar para lograr la precisión de un contenido que genere eficacia en este proceso.

Una concepción puede ser entendida como "un sistema de ideas, conceptos y representaciones sobre un aspecto de la realidad o toda ella, abarcando desde las filosóficas generales hasta las científico naturales" Entre los autores que se adscriben a esta posición están Ruiz. A, (1999); del Canto, C. (2000); Arturo Gayle (2005) y Silvia Navarro (2006), citados por Valle, 2010. 
La concepción para fundamentar la precisión del contenido con el cual se satisface el objetivo del diseño y rediseño de una carrera, debe reflejar conceptos, principios, leyes, condiciones y categorías que parten de la normativa que establece la LOES (2010) y los mecanismos establecidos por el Consejo de Educación Superior, Consejo de Evaluación, Acreditación y Aseguramiento de la Calidad de la Educación Superior (CEAACES) y el Régimen Académico de Ecuador, que es la demanda. A ello se unen las exigencias expresadas en el modelo de formación por competencias de la UISEK y las que adoptan la carrera.

Valoremos en síntesis cada subsistema presentado en la Figura 3.

\section{El proceso profesional - la necesidad social.-}

Del conocimiento que se tenga de la profesión, sus problemas profesionales, su proceso profesional, dependerá determinar sus perspectivas de desarrollo, porque ello decide la actualidad y la necesidad de la existencia de cada carrera para y por el desarrollo socioeconómico de un contexto.

No importa que la profesión responda a tradiciones de siglos como puede ser la medicina, la abogacía, entre otros. No importa que sea una profesión emergente. En todos los casos es imprescindible crear los mecanismos para el estudio de las profesiones, mediante los cuales se profundice en las características de su evolución, de su existencia en la vida. De este proceso se alcanza la demanda de la profesión en términos de la ciencia, la tecnología, de desempeño profesional.

Las carreras existen porque existe una profesión que requiere de un tipo de profesional para que resuelva sus problemas profesionales dentro de un proceso profesional determinado. He allí la clave que garantiza formar profesionales con respuesta laboral en la cantidad y calidad demandadas; he allí la clave que argumenta la necesidad de conocer con alta precisión la profesión.

Conocer la profesión que establece el diseño de una carrera y su sistemático rediseño, es posible mediante el trabajo de comisiones de expertos que integran docentes de experiencia universitaria y otros agentes externos del sector de la producción y los servicios, los que estudian las características de la profesión.

Los elementos descritos conforman el perfil del profesional, es decir, determinan los aspectos más generales y trascendentes que deben ser incorporados al proceso de formación de los profesionales con una intencionalidad formativa que debe incidir de forma directa en el desempeño profesional y social. Esta significación social cuando se tiene en consideración las características de la formación universitaria ha de ser universal y a la vez contextual, que es lo que le ofrece mirada comprometida con una realidad social y profesional en su contexto.

Del conocimiento que se tenga de la sociedad, su demanda acerca de las cualidades profesionales que han de caracterizar a sus egresados universitarios, expresadas en sus aspectos educativos y sintetizados en lo formativo, lo valorativo, lo estético y lo ético, es lo que hace que se precise el carácter pedagógico del rediseño de la carrera. 
Lo anterior satisface una concreción de la pertinencia con la cual debe diseñarse y rediseñarse la carrera, a partir de la realidad que se establece entre lo instructivo que demanda la profesión, así como lo educativo y desarrollador que exige la sociedad.

El graduado ha de ser un portador del dominio de la profesión para poder resolver los problemas presentes en dicha profesión. Ha de dominar la esencia y no el fenómeno del proceso profesional real en el cual debe insertarse en la vida profesional, pero debe ser también un profesional comprometido social y profesionalmente, flexible y trascendente que responda con calidad a las necesidades sociales cuando se desempeña en el objeto de la profesión.

La demanda social viene expresada dentro del marco normativo y de evaluación para el análisis de pertinencia, pero no debe ser una reproducción de esas normativas, debe ser una respuesta de la carrera a esas normativas. Desde este análisis se alcanza el conocimiento y la precisión de la demanda laboral basada en indicadores reales; la demanda del tipo de ciudadano que aspira formar el país, basada en las aspiraciones de desarrollo social.

El estudio de la necesidad social, expresado dentro del marco normativo y de evaluación para el análisis de pertinencia, cuando se vincula a las exigencias de la profesión, constituye en la relación tríadica expresada, cédula de la estructura del proceso, que desde un enfoque holográfico representa la igualdad presente en la demanda ecuatoriana para todas las universidades.

\section{El proceso de formación profesional.-}

A la igualdad de la demanda se contrapone la diferencia de cada universidad a través del proceso formativo que considera oportuno, suficiente y necesario para cumplir con su misión social.

Para nuestro caso, la diferencia radica en que cada proceso formativo expresa una nueva cualidad, que lo distingue. Justamente su igualdad y su diferencia es lo que permite la relación contradictoria, dialéctica cuya solución es la fuente del desarrollo del objeto.

Decididamente, en la actualidad, el proceso de formación profesional no es una respuesta integral e intencionada al proceso profesional y no marca conscientemente la diferencia. Allí está la clave de las insatisfacciones del sector productivo y de los servicios.

La idea de la pertinencia es contribuir a colocar el proceso formativo de las carreras en calidad de oferta formativa para que sus egresados respondan al proceso profesional de su profesión.

En la Figura 4 se puede sintetizar la interpretación que se alcanza en la UISEK para establecer su proceso formativo en todas y cada una de sus carreras.

El proceso formativo es una respuesta al proceso profesional. Desde sus procesos sustantivos el vínculo que se establece con el proceso profesional, logra que: 
- El proceso académico no sea escolástico, dogmático, porque promueve un currículo que atiende a los criterios existentes de la ciencia y sea profesionalizante. Por otra parte permite acercar a los estudiantes a la profesión en la cual se preparan y su desempeño se ajusta a saberes significativos.

- Respecto al proceso sustantivo laboral, el vínculo señalado facilita que se despliegue un currículo donde se desarrollan habilidades profesionales imposibles de reproducir en las universidades.

- En atención el proceso investigativo garantiza el desarrollo de la creatividad, porque los estudiantes aplican procesos investigativos cuyos métodos científicos son propios de condiciones reales de trabajo, de respuesta a necesidades reales.

Estos tres elementos constituyen en sí la esencia de la oferta formativa.

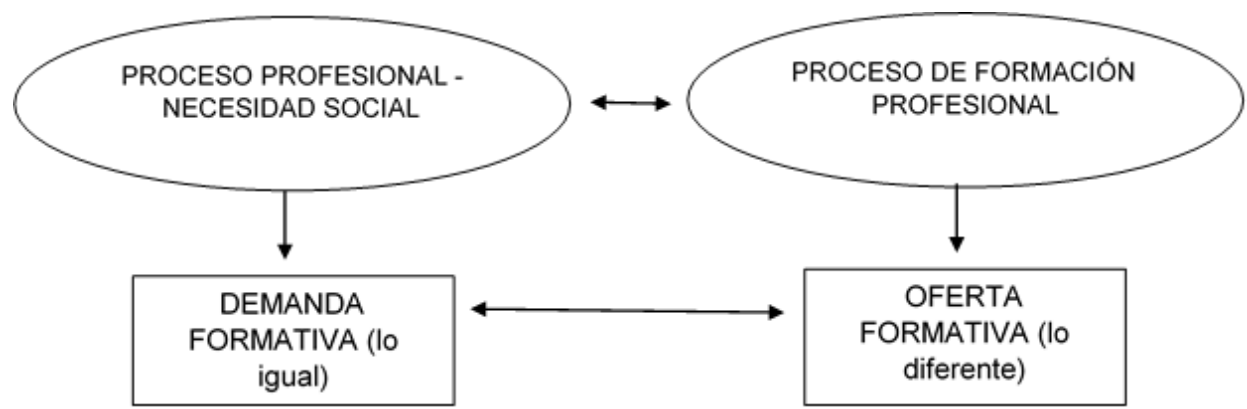

Figura 4. Síntesis de la relación de la oferta formativa de las carreras basadas en el proceso de formación profesional.

Llegar a una determinación de cómo debe ser el proceso de formación profesional en una carrera, es encontrar, descubrir, la mejor relación entre la oferta de la carrera y la demanda externa. Es convertir entonces la oferta en demanda, para que el sector de la producción y los servicios sea un aliado y contribuya, a partir de esa demanda, al proceso formativo desde el proceso profesional.

Para estudiar el proceso de formación profesional debe partirse, primeramente, de conocer qué modelo pedagógico y didáctico sustenta la universidad desde donde se diseña o rediseña la carrera. Como respuesta al proceso profesional y necesidad social permite precisar la aspiración de la oferta que hace la universidad en general y la carrera en particular a la sociedad. Sin embargo, el proceso profesional evoluciona con inusitada rapidez y las necesidades sociales crecen permanentemente. Hace falta pues un subsistema que regule esta contradicción.

\section{El proceso de pertinencia.-}

El principio de la pertinencia como se ha explicado establece un marco lógico que favorece conducir el diseño y rediseño de las carreras al cumplimiento de la primera Ley de Universidades. El proceso de pertinencia, en el diseño y rediseño de carreras, es una sucesión de etapas que establecen una mediación entre el proceso profesional y el proceso de formación profesional. Lo que es pertinente hoy, no lo será mañana, por lo que este principio favorecerá tanto la formación de lo conocido, como la perspectiva de formación de lo que se descubra como necesidad. 
Atendiendo a la relación tríadica (Figura 3), el tercer subsistema es el desarrollo del proceso de pertinencia que establece el vínculo entre los dos subsistemas contradictorios que representan lo igual y lo diferente; que posibilita un puente entre uno y otro que, al ser más dinámico que los otros dos, va modificándose cuantitativamente y posibilita el cambio cualitativo del diseño y rediseño de las carreras en un momento determinado.

El subsistema proceso de pertinencia, es portador al mismo tiempo de lo igual y lo diferente, mediando entre uno y otro, en correspondencia con las condiciones que se vayan presentando, es la solución de la contradicción, implica el desarrollo del proceso de diseño y rediseño de las carreras, su movimiento.

Como subsistema mediador, es portador de la característica del desarrollo de la demanda que establece el proceso profesional y de la oferta que promueve el proceso de formación profesional. En la actualidad, el proceso de pertinencia exige que ambos procesos (lo igual y lo diferente) evolucionen y se retroalimenten de la planificación nacional, del régimen de desarrollo nacional, de la prospectiva de desarrollo científico, humanístico y tecnológico mundial, así como de la diversidad cultural ecuatoriana.

De esta manera, el estudio del proceso de pertinencia, genera alcanzar una nueva cualidad en el sistema de relaciones que se devela entre el sector de la producción y los servicios que es donde se desarrollan las profesiones y las universidades, que es donde tiene lugar la formación de los estudiantes. Esta nueva cualidad fundamenta que el diseño y rediseño de las carreras sea pertinente.

\section{Conclusiones}

La interpretación que ha realizado la UISEK sobre la metodología de diseño y rediseño de sus carreras que establece el Consejo de Educación Superior ecuatoriano (2015), derivó en el establecimiento de una concepción teórica institucional acerca de cómo demostrar la asunción del principio de la pertinencia en los contenidos que lo argumentan. Esta concepción se concretó en el sistema de relaciones entre el proceso profesional, el proceso de formación profesional y el proceso de pertinencia universitaria, lo que pudo sintetizarse en la cualidad pertinente de una carrera universitaria.

\section{Bibliografía}

Álvarez, C. Epistemología del Caos. Grupo editorial Kipus. Primera edición. Cochabamba. Bolivia, 2004.

Arbizu Echávarri, F. M. La Formación Profesional Específica. Claves para el desarrollo curricular. Grupo Santillana de Ediciones. Madrid. p.22-29, 1998.

Barrios, E.A. y Delgado, J.M. Las competencias profesionales del docente universitario, el modelo educativo de la Universidad Internacional SEK_QUITO y el desempeño profesional 
docente, de la autoría de Vol. VI, no. 2, abril-junio de 2015. Revista Didasc@lia: Didáctica y Educación. ISSN 2224-2643.

Colectivo de autores. Proyecto FORCOM. Universidad Pedagógica "Frank País García" Santiago de Cuba. Material impreso, 2005.

Coll, C. Psicología y Currículum: Una aproximación psicopedagógica a la elaboración del currículum escolar. Editorial Paidós México, 1987.

Consejo de Educación Superior. Instructivo el reglamento de presentación y aprobación de proyectos de carreras y programas de grado y postgrado de las universidades y escuelas politécnicas. República del Ecuador, 2015. http://www.ces.gob.ec/gaceta-oficial/actas-yresoluciones/sesiones extraordinarias/2015. (Consultado 9 de septiembre de 2015).

Consejo de Educación Superior. Reglamento de Régimen Académico. República del Ecuador, 2013. http://www.ces.gob.ec/2012-10-29-08-51-25/2012-10-29-08-51-54. (Consultado 12 de septiembre de 2015).

Fuentes, H. Formación por competencias en la concepción de la universidad humana cultural una propuesta desde la Universidad Estatal de Bolívar. Guaranda, Provincia de Bolívar. Material impreso, 2010.

Fuentes. H. y Valiente, I. Dinámica del proceso docente educativo de la Educación Superior. Centro de estudios de Educación Superior "Manuel F. Gran”. Universidad de Oriente. Santiago de Cuba. Material impreso, 1998.

Ley Orgánica de Educación Superior. República de Ecuador, 2010. http://www.ces.gob.ec/descargas/ley-organica-deeducacionsuperior. (Consultado $3 \mathrm{de}$ septiembre de 2015)

Marimón, J. A. Aproximación al modelo como resultado científico en Los resultados científicos como aportes de la investigación educativa. ISP Félix Varela. Villa Clara, 2005.

Portuondo, R. y Barrios E. El perfil del técnico profesional. En soporte electrónico. p. 1 - 14,2004.

Universidad Internacional SEK. Modelo educativo basado en competencias de formación integral. Material impreso. Quito. Ecuador, 2014.

Valle Lima, A. La investigación pedagógica: otra mirada. Ciudad de La Habana. Material digital, 2010. 